\title{
Características de $\alpha$-acetolactato sintetasa y producción de diacetilo por Enterococcus faecium ETw7 y Enterococcus faecalis ETw23
}

\section{Characteristics of $\alpha$-acetolactate synthase and diacetyl production by Enterococcus faecium ETw7 and Enterococcus faecalis ETw23

\author{
Marisol Vallejo', Emilio Marguet ${ }^{1}$ y Valeria Etchechoury ${ }^{1}$
}

1 Facultad de Ciencias Naturales, Universidad Nacional de la Patagonia. Sede Trelen Roca 115 (9100) Trelew, Chubut - Argentina.

E mail Marisol Vallejo: soltrelew@yahoo.com.ar E mail Emilio Marguet:

\section{Resumen}

El diacetilo es un compuesto aromático esencial en productos lácteos fermentados como el queso. En este trabajo se estudiaron características cinéticas y bioquímicas de la $\alpha$-acetolactato sintetasa ( $\alpha$-ALS) y su influencia en la producción de diacetilo en Enterococcus faecium ETw7 y Enteroccoccus faecalis ETw23. En ambos casos, los parámetros cinéticos revelaron una baja afinidad por el piruvato, como ha sido descrito en otras bacterias ácido lácticas. E. faecium ETw7 desarrolló la máxima actividad enzimática a pH 5,8-6,2 y $40^{\circ} \mathrm{C}$, sin embargo bajo las condiciones de maduración de quesos ( $\mathrm{pH} 5,0$ y $15^{\circ} \mathrm{C}$ ) la actividad remanente fue baja. La $\alpha-A L S$ de $E$. faecalis ETw23 mostró la máxima actividad al pH de maduración, la temperatura óptima fue determinada a $40^{\circ} \mathrm{C}$ y la actividad remanente a $15^{\circ} \mathrm{C}$ fue aproximadamente el $30 \%$ de la máxima. El crecimiento y la producción de diacetilo fue estudiada en el medio De Man-Rogosa-Sharpe (MRS) y MRS suplementado con citrato (MRScit). La tasa de crecimiento de E. faecium ETw7 fue comparable en ambos medios, pero se observó un aumento de la biomasa en MRScit. En el caso de E. faecalis ETw23 se logró una mayor tasa de crecimiento entre las 6 y $10 \mathrm{~h}$, y una mayor biomasa en MRScit. Después de $24 \mathrm{~h}$ de crecimiento E. faecium ETw7 alcanzó un nivel de 20,4 $\mu \mathrm{M}$ de diacetilo en MRS y 26,1 $\mu \mathrm{M}$ en MRScit, mientras que E. faecalis ETw23 logró niveles de 41,8 $\mu \mathrm{M}$ y 61,7 $\mu \mathrm{M}$, respectivamente. Los resultados de este estudio sugieren que E. faecalis ETw23 puede contribuir en el desarrollo de aromas en quesos a través de su rol en la producción de diacetilo.

Palabras claves: $\alpha$-acetolactato sintetasa, citrato, diacetilo, Enterococcus, queso.

\section{Abstract}

Diacetyl is an essential flavor compound in fermented dairy products such as cheese. In this work kinetic and biochemical characteristics of $\alpha$-acetolactate sinthase ( $\alpha$-ALS) and its influence on the formation of diacetyl were studied in Enterococcus faecium ETw7 and Enteroccoccus faecalis ETw23. In both cases, the kinetic parameters revealed a low affinity for piruvate, as has been described in other lactic acid bacteria. E. faecium ETw7 displayed its maximal enzimatic activity at $\mathrm{pH} 5.8-6.2$ and $40^{\circ} \mathrm{C}$, however under cheese ripening condition $\left(\mathrm{pH} 5.0\right.$ and $\left.15^{\circ} \mathrm{C}\right)$ the remaining activity was low. $\alpha$-ALS from $E$. faecalis ETw23 showed its maximal activity at ripening $\mathrm{pH}$, the optimun temperature was determined at $40^{\circ} \mathrm{C}$ and the remaining activity at $15^{\circ} \mathrm{C}$ was about $30 \%$ of its maximal one. The growth and diacetyl formation by both strains were studied in De Man-Rogosa-Sharpe medium (MRS) and MRS supplemented with citrate (MRScit). In both medium the growth rate of $E$. faecium ETw7 was comparable but an enhancement in biomass was observed in MRScit. In the case of $E$. faecalis ETw23 a higher growth rate, between $6 \mathrm{~h}$ and $10 \mathrm{~h}$, and a higher biomass were achieved in MRScit. After 24 h of growth, E. faecium ETw7 reached a level of $20.4 \mu \mathrm{M}$ of diacetyl in MRS and $26.1 \mu \mathrm{M}$ in MRScit, while $E$. faecalis ETw23 achieved levels of $41.8 \mu \mathrm{M}$ and $61.7 \mu \mathrm{M}$, respectively. The results of the study suggest that $E$. faecalis ETw23 may contribute to flavor development in cheese through its role in diacetyl production.

Keywords: $\alpha$-acetolactate synthase, citrate, diacetyl, Enterococcus, cheese

\section{Introducción}

El diacetilo es considerado uno de los compuestos más influyentes en la generación de aromas característicos en productos lácteos fermentados como manteca, leches cultivadas y quesos (McSweeney y Sousa, 2000). Las bacterias ácido lácticas (BAL), utilizadas en la elaboración de este tipo de alimentos, son fermentadoras obligadas que producen principalmente ácido láctico a partir de azúcares y en las que la producción de diacetilo constituye una propiedad fisiológica de interés industrial.

Durante mucho tiempo se especuló que el diacetilo era el producto de una reacción enzimática en la que el $\alpha$-acetolactato $(\alpha-\mathrm{AL})$ actuaba como sustrato, sin embargo nunca se pudo identificar y/o purificar una enzima que llevara a cabo la reacción descrita. Ramos et al. (1994), demostraron que el diacetilo provenía de una reacción espontánea que se originaba a partir de la descarboxilación oxidativa del $\alpha-A L$. Éste es producido por la condensación de dos moléculas de piruvato por la acción de la $\alpha$-acetolactato sintetasa ( $\alpha-A L S)$, que constituye una enzima clave en la generación de compuestos aromáticos.

Las BAL poseen una $\alpha$-ALS con una baja afinidad por el sustrato (50 mM) (Hugenholtz, 1993; Marugg et al., 1994) y en consecuencia la posibilidad de producir $\alpha-A L$ y posteriormente diacetilo, sólo queda reducida a aquellas especies capaces de utilizar citrato como fuente de carbono, cuyo metabolismo contribuye a aumentar la concentración de piruvato intracelular (Cogan, 1987; Palles et al., 1998; Kimono et al., 1999).

Con el objeto de producir diacetilo en la elaboración industrial de quesos, se utilizan fermentos en los que se incluyen cepas con la habilidad de transportar citrato a través de la membrana, propiedad limitada solamente a algunas especies del género Leuconostoc y Lactococcus lactis subsp. lactis biovar diacetylactis (Schmitt y Divies, 1992; Boumerdassi et al., 1997).

Las BAL del fermento se encuentran al comienzo de la maduración de quesos en un alto número, que luego comienza a declinar a medida que el proceso avanza. En las últimas etapas de la maduración, la flora adjunta, originaria del ambiente de las queserías o de la leche, resulta dominante en la masa casearia y no obstante estar constituida por diferentes microorganismos, se destacan por su elevada población, las cepas del género Enterococcus (Sarantinopoulos et al., 2001a).

Algunos trabajos pioneros mostraron que los miembros de este género tenían la capacidad de formar acetato y formato a partir de citrato (Campbell y Gunsalus, 1944; Coventry et al., 1978). Sin embargo, otros estudios demostraron que los Enterococcus pueden metabolizar citrato en presencia de lactosa y en consecuencia producir una concentración suficiente de piruvato que 
permite su utilización para la síntesis de $\alpha-\mathrm{AL}$ (Sarantinopoulos et al., 2001b). Esta característica metabólica despertó un particular interés debido a la posibilidad de utilizar cepas del género Enterococcus como fermentos adjuntos en la elaboración de quesos (Centeno et al., 1999; Giraffa, 2003).

En el presente trabajo se determinaron las características fisicoquímicas y cinéticas de $\alpha$-acetolactato sintetasa de Enterococcus faecium ETw7 y Enterococcus faecalis ETw23 con el objeto de establecer la influencia que ejercen en la producción de diacetilo. Se analizaron también la actividad de la enzima y la producción de diacetilo en caldo De Man-Rogosa-Sharpe (MRS) (De Man et al., 1960) y en caldo MRS suplementado con citrato.

\section{Materiales y métodos}

Las cepas de Enterococcus faecium ETw7 y Enterococcus faecalis ETw23 utilizadas en este estudio pertenecen a la colección de bacterias ácido lácticas de la Cátedra de Biología Celular y Molecular (Facultad de Ciencias Naturales, sede Trelew, Universidad Nacional de la Patagonia, Argentina). Ambas cepas se incubaron durante $18 \mathrm{~h}$ a $35^{\circ} \mathrm{C}$ en caldo MRS y caldo MRS suplementado con citrato de sodio (MRScit). Luego de este período se procedió a una centrifugación de $5000 \mathrm{~g}$ durante $5 \mathrm{~min}$. Las células obtenidas se trataron con cuentas de vidrio y vórtex durante 10 min con el objeto de liberar su contenido citoplasmático. Luego de este tratamiento se centrifugaron nuevamente los extractos celulares a $5000 \mathrm{~g}$ durante 5 minutos y se determinó la actividad enzimática de los sobrenadantes.

La actividad enzimática de los sobrenadantes se realizó en una mezcla de reacción que contenía buffer fosfato sódico $(\mathrm{pH}$ 7,$0 ; 0,1 \mathrm{M}$ ), cocarboxilasa $0,21 \mathrm{M}$ y piruvato de sodio $0,8 \mathrm{M}$ (Boumerdassi et al., 1997). Luego de una incubación de 30

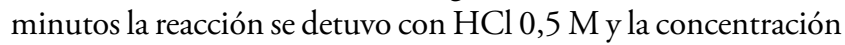
de diacetilo producido a partir de piruvato se determinó por el método colorimétrico de Westerfeld (Westerfeld, 1945).

La actividad enzimática de los sobrenadantes se ensayó desde $\mathrm{pH}$ 5,0 hasta 8,6 y se utilizó buffer acetato $0,1 \mathrm{M}$ en el rango 5,0-6,4; buffer fosfato $0,1 \mathrm{M}$ en el rango 6,6-7,6 y buffer Tris $0,1 \mathrm{M}$ en el rango 7,8-8,6.

La actividad enzimática de los sobrenadantes se ensayó, a pH óptimo, a las temperaturas de 15, 20, 25, 30, 35, 40 y $45^{\circ} \mathrm{C}$.

El $K_{m}$ y la Velocidad Máxima $\left(V_{m a x}\right)$ de la reacción enzimática se determinaron en condiciones óptimas de $\mathrm{pH}$ y temperatura

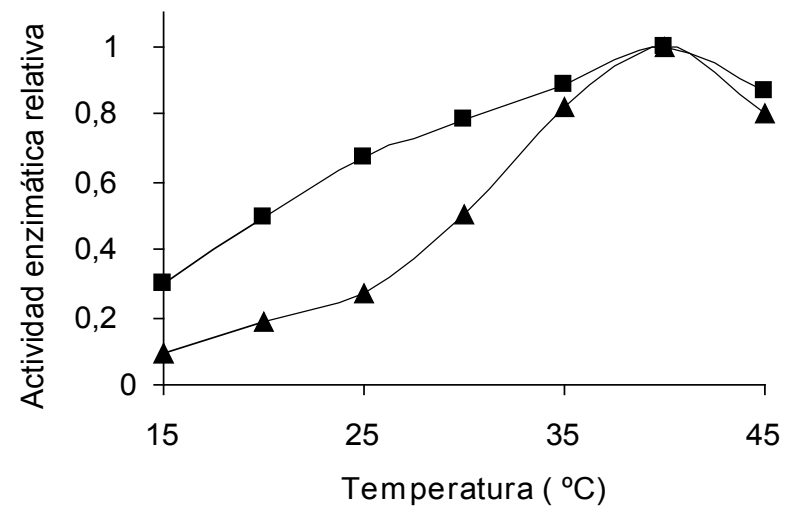

Figura 1. Actividad enzimática relativa de $\alpha$-acetolactato sintetasa de Enterococcus faecium ETw7 ( $\mathbf{\Delta}$ ) y Enterococcus faecalis ETw23 (घ) en función de la temperatura. y utilizando la gráfica de Lineweaver-Burk (Philips, 1994). Los cálculos de los parámetros cinéticos se realizaron en base a la ecuación de la recta obtenida en la línea de tendencia. La $V_{\max }$ se expresó como la cantidad de $\mu$ moles de diacetilo producidos por minuto por miligramo de proteína ( $\mu$ moles/min.mg).

La concentración de proteínas de los sobrenadantes se determinó por el método de Bradford utilizando albúmina bovina como estándar (Bradford, 1976).

Ambas cepas se cultivaron en caldo MRS (concentración de citrato 0,2\%), y caldo MRScit (concentración final de citrato $0,4 \%$ ) en las condiciones anteriormente descritas. Cada 2 horas se tomaron muestras y se leyeron sus densidades ópticas en espectrofotómetro a $600 \mathrm{~nm}\left(\mathrm{OD}_{600}\right)$, hasta completar las 24 horas. El diacetilo de las muestras se determinó por el método de Westerfeld (Westerfeld, 1945).

\section{Resultados y discusión}

\section{Temperatura y pH óptimos}

Se han reportado numerosos trabajos donde se destaca la influencia del metabolismo de los enterococos durante la elaboración de quesos (Centeno et al., 1999; Giraffa, 2003). En consecuencia resulta de interés investigar el comportamiento de enzimas involucradas en el desarrollo de aromas y sabores característicos, especialmente bajo las condiciones de maduración en cámara ( $\mathrm{pH} 5,0$ y $15^{\circ} \mathrm{C}$ ). En este caso en particular se estudiaron las cepas E. faecium ETw7 y E. faecalis ETw23, seleccionadas por la alta producción de diacetilo.

En al Figura 1 se observa que la temperatura óptima de la actividad de $\alpha$-ALS de ambas cepas fue $40^{\circ} \mathrm{C}$. Sin embargo se observan diferencias cuando los ensayos se realizan a $15^{\circ} \mathrm{C}$ : $E$. faecium ETw7 exhibe valores por debajo del 10\% de la actividad obtenida a la temperatura óptima, mientras E. faecalis ETw23 la mantiene por encima del $30 \%$.

En la Figura 2 podemos apreciar los resultados de la actividad enzimática en función del $\mathrm{pH}$. En el rango 5,8-6,2 se determinó la máxima actividad para E. faecium ETw7, observándose un considerable descenso cuando los ensayos se llevaron a cabo a $\mathrm{pH}$ 5,0. Efaecalis ETw23 exhibió su máxima actividad a pH 5,0 coincidiendo en este caso con el valor que se logra en la masa del queso durante su maduración en cámara.

Tomando en consideración ambos parámetros, temperatura y $\mathrm{pH}$, E. faecium ETw7 muestra un marcado descenso de la ac-

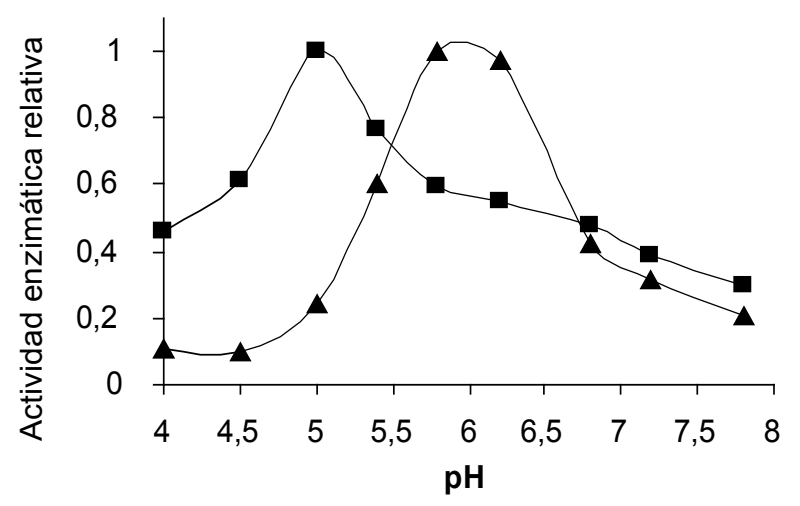

Figura 2. Actividad enzimática relativa de $\alpha$-acetolactato sintetasa de Enterococcus faecium ETw7 ( $\mathbf{\Delta}$ ) y Enterococcus faecalis ETw23 (匹) en función del pH. 
tividad de $\alpha$-ALS en las condiciones de maduración, mientras que E. faecalis ETw23 presenta características fisicoquímicas, que le permiten generar mayores concentraciones de $\alpha-\mathrm{AL}$ y metabolitos implicados en la producción de aromas, influyendo en la calidad del producto final.

\section{Determinación de parámetros cinéticos}

Los valores de $K_{m}$ y $V_{\text {max }}$ de $\alpha$-ALS determinados según la gráfica inversa de Lineweaver-Burk fueron $53 \mathrm{mM}$ y 17,06 $\mu$ moles/ min.mg para E. faecium ETw7 y 29 mM y 36,49 $\mu$ moles/min. mg para E. faecalis ETw23, respectivamente. En ambos casos se observa una baja afinidad por el sustrato, una característica que ha sido descrita en otras cepas de BAL (Hugenholtz, 1993; Marugg et al., 1994). También los valores de $V_{\text {max }}$ son comparables con los encontrados en anteriores trabajos (Sarantinopoulos et al., 2003).

El $\alpha-\mathrm{AL}$ es producido por las BAL sólo cuando se logra acumular piruvato dentro de las células en altas concentraciones. En BAL homofermentativas, como es el caso del género Enterococcus, el piruvato es un intermediario común formado durante el metabolismo de azúcares y citrato. Cuando estas bacterias tienen un azúcar como única fuente de carbono la mayor concentración de piruvato es convertido en lactato gracias a la acción de la Láctico deshidrogenasa (LDH) que exhibe una gran afinidad por el sustrato. En cambio cuando el medio contiene citrato se elevan las concentraciones de piruvato permitiendo que ejerzan su actividad catalítica enzimas que tienen más baja afinidad por el sustrato que la LDH.

Los valores de $K_{m}$ determinados en los casos estudiados, nos permite aseverar que $E$. faecium ETw7 solo tiene la posibilidad de generar $\alpha-A L$ cuando la concentración intracelular de piruvato es superior a $53 \mathrm{mM}$ mientras que E. faecalis $\mathrm{ETw} 23$ requiere una concentración superior a $29 \mathrm{mM}$.

\section{Crecimiento y producción de diacetilo}

Las experiencias realizadas para determinar la influencia del citrato en el crecimiento y en la producción de diacetilo por las cepas estudiadas se llevaron a cabo durante $24 \mathrm{~h}$ en caldo MRS y caldo MRScit.

Como se observa en la Figura 3, E. faecium ETw7 llega a la fase estacionaria a las $10 \mathrm{~h}$ de incubación mientras que el medio suplementado con citrato alcanza esa condición a las $12 \mathrm{~h}$, lográndose en consecuencia una mayor biomasa.

No se observan diferencias significativas en las curvas de crecimiento hasta las $8 \mathrm{~h}$ cuando comparamos los 2 medios,

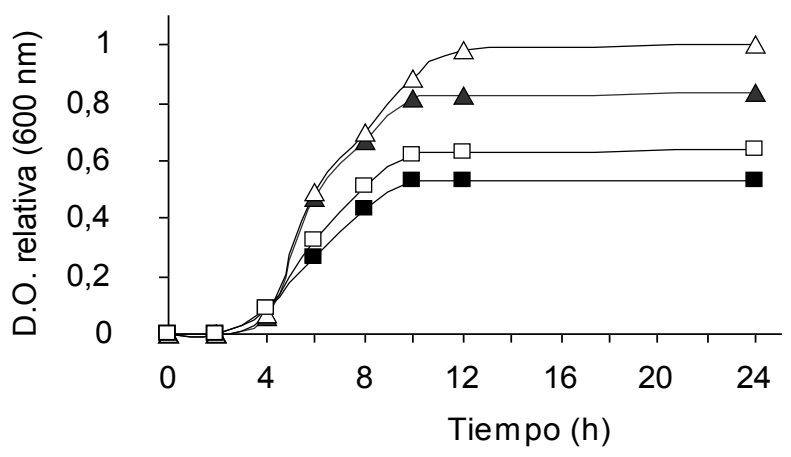

Figura 3. Crecimiento de Enterococcus faecium ETw7 en MRS ( $\Delta$ ) y en MRScit $(\Delta)$ y de Enterococcus faecalis ETw23 en MRS (a) y en MRScit ( $\square)$. coincidiendo con las experiencias que indican que una mayor concentración de citrato no influye en el aumento de la velocidad de duplicación, pero contribuye para alcanzar una mayor población final (Sarantinopoulos et al., 2003). La Figura 4 indica que, no obstante lograrse una mayor producción de diacetilo a las 6 h de incubación en MRScit, la diferencia de concentración entre ambos medios permanece sin modificaciones apreciables hasta las 24 h. Las Figuras 2 y 3 permiten concluir que en el caso de $E$. faecium ETw7 el citrato es utilizado en forma eficiente para lograr energía (ATP) por la vía de la acetato-quinasa, en detrimento de la recuperación de $\mathrm{NAD}^{+}$que se logra a través del camino metabólico iniciado por la $\alpha$-ALS (Sarantinopoulos et al., 2003; Vaningelgem et al., 2006).

En el caso de E. faecalis ETw23 se observa una mayor la velocidad de duplicación en el MRScit a partir de las 6 h de incubación, alcanzando ambos medios la fase estacionaria a las $10 \mathrm{~h}$ (Fig. 3). También en este caso se logra en el medio con mayor concentración de citrato una biomasa mayor, coincidiendo con lo descrito en trabajos previos (Sarantinopoulos et al., 2001b).

En ambas experiencias, con MRS o MRScit, es posible apreciar que las poblaciones finales en la fase estacionaria de E. faecium ETw7 son mayores que las alcanzadas por E. faecalis ETw23.

En la Figura 4 se observan diferencias significativas en la producción de diacetilo entre ambas cepas. Mientras que E. faecalis ETw23 alcanzó, luego de $24 \mathrm{~h}$ de incubación, concentraciones de 61,7 $\mu \mathrm{M}$ en caldo MRScit y 41,8 $\mu \mathrm{M}$ en MRS, E. faecium ETw7 logró concentraciones de $26,1 \mu \mathrm{M}$ y $20,4 \mu \mathrm{M}$, respectivamente.

La curvas de crecimiento y de producción de diacetilo permiten observar los diferentes comportamientos de las cepas estudiadas: mientras E. faecium ETw7 logró obtener mayores niveles de energía a partir del piruvato derivado del citrato y en consecuencia una mayor biomasa; E. faecalis ETw23 metabolizó el piruvato a través de la $\alpha-A L S$ con el propósito de obtener mayores niveles de $\mathrm{NAD}^{+}$, generando mayores concentraciones de diacetilo.

\section{Actividad de $\alpha$-ALS}

La $\alpha$-ALS es activa sólo en presencia de altas concentraciones de piruvato (Snoep et al., 1992; Marugg et al., 1994) situación que solo es posible cuando hay citrato disponible en el medio y la célula posee los mecanismos para transportarlo a través de la membrana.

En la Figura 5 se pueden observar las variaciones de la actividad de $\alpha$-ALS en las condiciones previamente descritas. En ambos

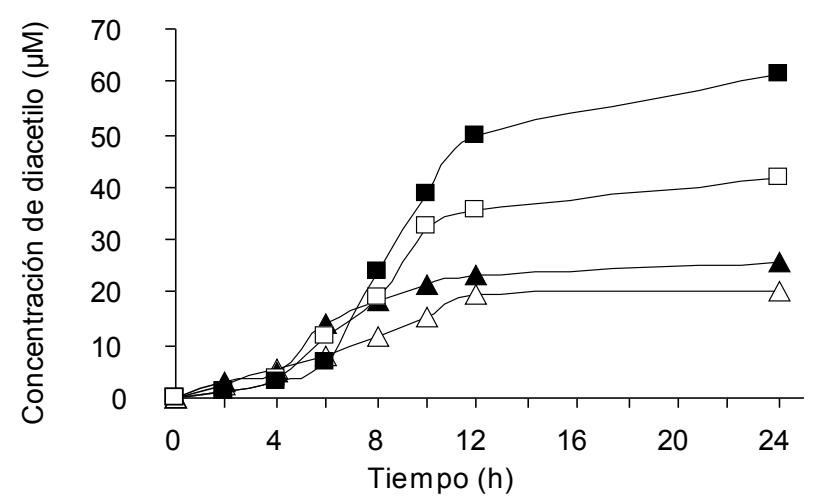

Figura 4. Producción de diacetilo de Enterococcus faecium ETw7 en MRS $(\boldsymbol{\Delta})$ y en MRScit $(\Delta)$ y de Enterococcus faecalis ETw23 en MRS ( $\bullet$ ) y en MRScit (口). 


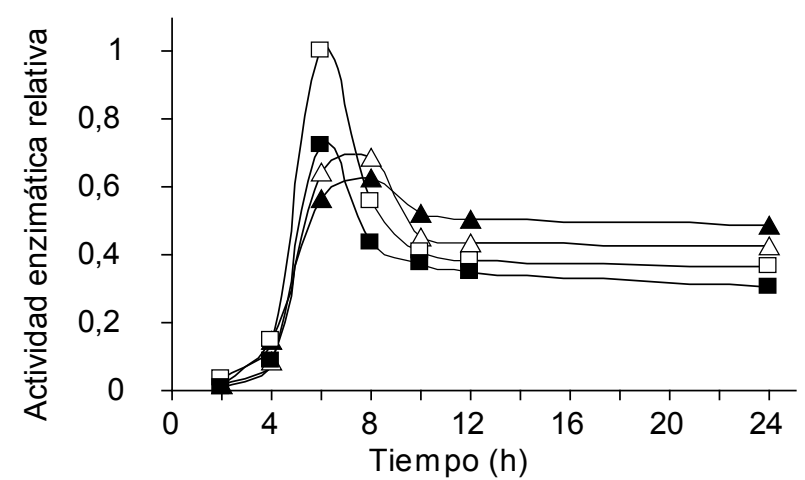

Figura 5. Actividad enzimática relativa de $\alpha$-acetolactato sintetasa de Enterococcus faecium ETw7 en MRS $(\boldsymbol{\Delta})$ y en MRScit $(\Delta)$ y de Enterococcus faecalis ETw23 en MRS (₫) y en MRScit ( $\square$ ).

medios, MRS y MRScit, E. faecium ETw7 exhibió la máxima actividad entre las 6 y $8 \mathrm{~h}$ de incubación mientras que $E$. faecalis ETw23 presentó un máximo a las $6 \mathrm{~h}$. Luego del período de máxima actividad se observa, en todos los casos, un marcado descenso que indicaría una baja estabilidad de la enzima.

Ambas cepas mostraron las más altas actividades enzimáticas en los medios que contenían menor concentración de citrato, fenómeno paradójico pero que se relaciona con las características de la Citrato permeasa, cuya máxima velocidad de transporte ocurre en un rango de $\mathrm{pH}$ entre 4,0 y 5,5 (Vaningelgem, 2006). El citrato actúa como un buffer y en consecuencia a mayores concentraciones más lentamente declina el $\mathrm{pH}$.

\section{Conclusiones}

La posibilidad de utilizar cepas de Enterococcus en la industria de la alimentación, especialmente en la elaboración de productos lácteos (Centeno et al., 1999; Giraffa, 2003), ha provocado el desarrollo de investigaciones tendientes a estudiar características metabólicas de interés biotecnológico.

En este trabajo se estudiaron características de la $\alpha-A L S$ y la producción de diacetilo bajo diferentes condiciones de 2 cepas del género Enterococcus, con el propósito de evaluar la posibilidad de incluirlos como fermentos adjuntos en la elaboración de quesos.

E. faecalis ETw23, además de generar mayores concentraciones de diacetilo que $E$. faecium $\mathrm{ETw} 7$ en las experiencias realizadas, exhibió características fisicoquímicas y cinéticas de $\alpha$-ALS que demuestran una alta actividad enzimática remanente en la condiciones de maduración de quesos.

En el futuro se deberán realizar estudios experimentales para demostrar que la inclusión de $E$. faecalis ETw23 en la elaboración de quesos tiene influencia efectiva en la generación de diacetilo y en la calidad final del producto.

\section{Literatura citada}

Boumerdassi, H.; C. Monnet, M. Desmazeaud and G. Corrieu. 1997. Isolation and properties of Lactococcus lactis subsp. lactis biovar diacetylactis CNRZ 483 mutants producing diacetyl and acetoin from glucose. Appl.Environ.Microbiol. 63: 2293-2299.

Bradford, M.M. 1976. A rapid and sensitive method for the quantitation of microgram quantities of protein utilizing the principle of protein-dye binding. Anal. Biochem. 72: 248-254.

Campbell, J.J.R. and I.C. Gunsalus. 1944. Citric acid fermentation by streptococci and lactobacilli. J. Bacteriol. 48: 71-76.
Centeno, J.A.; S. Menéndez; M.A. Hermida and J.L. RodriguezOtero. 1999. Effects of the addition of Enterococcus faecalis in Cebreiro cheese manufacture. Int. J. Food Microbiol. 48: 97- 101.

Cogan, T.M. 1987. Co-metabolism of citrate and glucose by Leuconostoc spp.: effects on growth, substrates and products. J. Appl. Bacteriol. 63: 551-558.

Coventry, M.J.; A.J. Hillier and G.R. Jago. 1978. The metabolism of pyruvate and citrate in the thermoduric cheese starter Streptococcus faecium (Streptococcus durans). Aust. J. Dairy Technol. 33: 148-154.

De Man J.; M. Rogosa and M.E. Sharpe. 1960. A medium for the cultivation of lactobacilli. J. Appl. Bact. 23: 130-135.

Giraffa, G. 2003. Functionality of enterococci in dairy products. Int. J. Food Microbiol. 88: 215-222.

Hugenholtz, J. 1993. Citrate metabolism in lactic acid bacteria. FEMS Microbiol. Rev. 12: 165-178.

Kimoto, H.; M. Nomura and I. Suzuki. 1999. Growth energetics of Lactococcus lactis subsp. lactis biovar diacetylactis in cometabolism of citrate and glucose. Int. Dairy J. 9: 857-863.

Marugg, D.; D. Goelling; U. Stahl; A.M. Ledeboer; M.Y. Toonen; W.M. Verhue and C.T. Verrips. 1994. Identification and characterization of the alpha-acetolactate synthase gene from Lactococcus lactis subsp. lactis biovar diacetylactis. Appl. Environ. Microbiol. 60: 1390-1394

McSweeney, P. and J. Sousa. 2000. Biochemical pathway for the production of flavour compounds in cheese during ripening: A review. Lait. 80: 293-324.

Palles, T.; T. Beresford; S. Condon and T.M. Cogan. 1998. Citrate metabolism in Lactobacillus casei and Lactobacillus plantarum. J. Appl. Microbiol. 85: 147-154.

Philips, A.T.1994. Enzymatic activity. In: Methods for general and molecular bacteriology. P. Gerhardt, R. G. E. Murray, W. A. Wood, N. R. Krieg ed. American Society for Microbiology. Washington D. C., P.555-605.

Ramos, A.; N.K. Jordan; T. Cogan and H. Santos. $1994 .{ }^{13} \mathrm{C}$ nuclear magnetic resonance studies of citrate and glucose cometabolism in Lactococcus lactis. Appl. Environ. Microbiol. 60: 1739-1748.

Sarantinopoulos, P.; C. Andrighetto; M.D. Georgalaki; M.C. Rea; A. Lombardi; T.M. Cogan; G. Kalantzopoulos and E. Tsakalidou. 2001a. Biochemical properties of enterococci relevant to their technological performance. Int. Dairy J. 11: 621-647.

Sarantinopoulos, P.; G. Kalantzopoulos and E. Tsakalidou. $2001 \mathrm{~b}$. Citrate metabolism by Enterococcus faecalis FAIR-E 229. Appl. Environ. Microbiol. 67: 5482- 5487.

Sarantinopoulos, P.; L. Makrasa; F. Vaningelgemb; G. Kalantzopoulos; L. De Vuyst and E. Tsakalidou. 2003. Growth and energy generation by Enterococcus faecium FAIR-E 198 during citrate metabolism. Int. J. Food Microbiol. 84: 197-206.

Schmitt, P. and C. Divies. 1992. Origin of end-products from cometabolism of glucose and citrate by Leuconostoc mesenteroides subsp. cremoris. Appl. Microbiol. Biotechnol. 36: 679- 683 .

Snoep, J.L.; M.J. Teixeira de Mattos; M.J.C. Starrenburg and J. Hugenholtz. 1992. Isolation, characterization, and physiological role of the pyruvate dehydrogenase complex and a-acetolactate synthase of Lactococcus lactis subsp. lactis bv. diacetylactis. J. Bacteriol. 174: 4838-4841.

Vaningelgem,F; V. Ghijsels; E. Tsakalidou and L. De Vuyst. 2006. Cometabolism of Citrate and Glucose by Enterococcus faecium FAIR-E 198 in the Absence of Cellular Growth. Appl. Environ. Microbiol. 72: 319-326

Westerfeld, W.W. 1945. A colorimetric determination of blood acetoin. J. Biol. Chem. 161: 495- 502. 